\title{
Penfigoide Bolhoso Tratado com Sucesso com Rituximab
}

\section{Bullous Pemphigoid Successfully Treated with Rituximab}

\begin{abstract}
Nuno SILVA $\rrbracket^{1}$, Andreia COSTA ${ }^{1}$, Fernando SALVADOR ${ }^{1}$, Elisa SERRADEIRO ${ }^{1}$
Acta Med Port 2017 Mar;30(3):243-246 - https://doi.org/10.20344/amp.7812

RESUMO

O penfigoide bolhoso é uma doença autoimune caracterizada pela formação de bolhas subepidérmicas. Pode ser autolimitada mas quando necessário o tratamento passa pelo uso de corticoides, imunossupressores, e em última linha agentes biológicos. Apresentase o caso de um doente de 37 anos com penfigoide bolhoso com uma forma de apresentação grave e refratária. Após cerca de um mês de tratamento com vários fármacos de primeira linha, por ausência de resposta, optou-se por iniciar rituximab com franca melhoria das lesões. Os autores pretendem realçar a utilidade e eficácia deste fármaco nesta patologia.
\end{abstract}

Palavras-chave: Penfigoide Bolhoso/tratamento; Rituximab

\section{ABSTRACT}

Bullous pemphigoid is an autoimmune disease characterized by subepidermal blister formation. Can be self-limiting but when necessary treatment includes the use of corticosteroids, immunosuppressants, and biological agents. We present the case of a 37 year old patient with a severe and refractory presentation of bullous pemphigoid. After about a month of treatment with various first line drugs and with no response, we chose to start rituximab and clear improvement of the lesions was observed. The authors aim to highlight the usefulness and effectiveness of this drug in this disease.

Keywords: Pemphigoid, Bullous/drug therapy; Rituximab

\section{INTRODUÇÃO}

O penfigoide bolhoso (PB) é uma doença autoimune caracterizada pela formação de bolhas na pele e, menos frequentemente, nas mucosas, mediada por anticorpos direcionados contra a membrana basal cutânea. Estima-se uma incidência, nos países desenvolvidos, de 0,2 a 3 casos por 100 mil habitantes por ano. Afeta igualmente indivíduos de ambos os sexos e é mais comum em idosos, mas também pode ocorrer em jovens e crianças. $O$ diagnóstico é feito com base em dados clínicos, analíticos e histopatológi$\cos .^{1} \mathrm{O}$ PB faz parte de um grupo de dermatoses bolhosas autoimunes que se podem classificar em intraepidérmicas e subepidérmicas conforme a localização da bolha. Deste grupo fazem parte o pênfigo vulgar, cujas bolhas, intraepidérmicas, são flácidas, frágeis e rompem-se rapidamente, formando erosões dolorosas, que sangram com facilidade. Por outro lado, o penfigoide bolhoso cursa com bolhas subepidérmicas e tensas. ${ }^{2} \mathrm{O}$ PB é normalmente autolimitado mas pode prolongar-se até cinco anos. Antes da introdução da corticoterapia a taxa de mortalidade podia chegar aos $24 \%{ }^{3}$

\section{CASO CLÍNICO}

Doente de 37 anos, sexo masculino, oligofrénico, seguido em consulta de psiquiatria, medicado com risperidona, quetiapina e zuclopentixol, referenciado ao serviço de urgência por lesões dérmicas bolhosas infetadas associado a febre com 13 dias de evolução. $O$ doente referia prurido mas negava dor. Sem história de introdução recente de fármacos. No exame físico apresentava-se hemodinamicamente estável, com pele com múltiplas lesões bolhosas dispersas por todo o corpo e algumas zonas com sinal de Nikolsky positivo, poupando as mucosas. Analiticamente apresentava de relevo 12700 eosinófilos/uL e proteína C reativa $6,3 \mathrm{mg} / \mathrm{dL}$. Iniciou tratamento com flucloxacilina, corticoide tópico, prednisolona (PDN) oral (1 mg/kg/dia) e, pela exuberância do quadro, e forte suspeita clínica de PB, também imunoglobulina endovenosa (IgIV). Realizou biópsia cutânea que revelou a presença de "vesícula subepidérmica e derme com leve infiltrado polimorfo com linfócitos, histiócitos e diversos eosinófilos", compatível com a hipótese diagnóstica. Posteriormente o estudo de imunofluorescência direta revelou a deposição linear na membrana basal de C3. O estudo imune foi negativo exceto para o anticorpo anti-BP180 (173 U/mL para um limite superior do normal de $9 \mathrm{U} / \mathrm{mL}$ ). Ao fim de três dias, por não haver melhoria clínica, adicionou-se azatioprina (AZA) $50 \mathrm{mg} / \mathrm{dia}$. Pela necessidade de cuidados diferenciados foi transferido temporariamente para uma unidade de queimados e depois admitido numa unidade de cuidados intermédios. Apesar de todas as medidas houve agravamento das lesões tendo-se então aumentado a dose de AZA para $150 \mathrm{mg} /$ dia e PDN para $1,5 \mathrm{mg} / \mathrm{kg} / \mathrm{dia}$ (90 mg). Realizou novo ciclo com IgIV uma semana depois. Ao fim de 40 dias de internamento sem melhoria clínica (Fig.s 1, 2) decidiu-se, em reunião de grupo de Doenças Autoimunes, iniciar rituximab. Efetuou quatro administrações semanais $\left(375 \mathrm{mg} / \mathrm{m}^{2}\right) \mathrm{com}$ excelente resposta (Fig. 3). Após a alta manteve seguimento em consulta externa com estabilidade clínica, apenas com lesões sequelares (MMPDAl actividade 0; dano 7) (Fig. 4) e diminuição dos títulos de anti-BP180 até $21,4 \mathrm{U} / \mathrm{mL}$.

1. Serviço de Medicina Interna. Unidade de Doenças Autoimunes. Centro Hospitalar de Trás-os-Montes e Alto Douro. Vila Real. Portugal.

$\triangle$ Autor correspondente: Nuno Silva. nunoreis13@hotmail.com

Recebido: 02 de maio de 2016 - Aceite: 23 de setembro de 2016 | Copyright $\odot$ Ordem dos Médicos 2017 


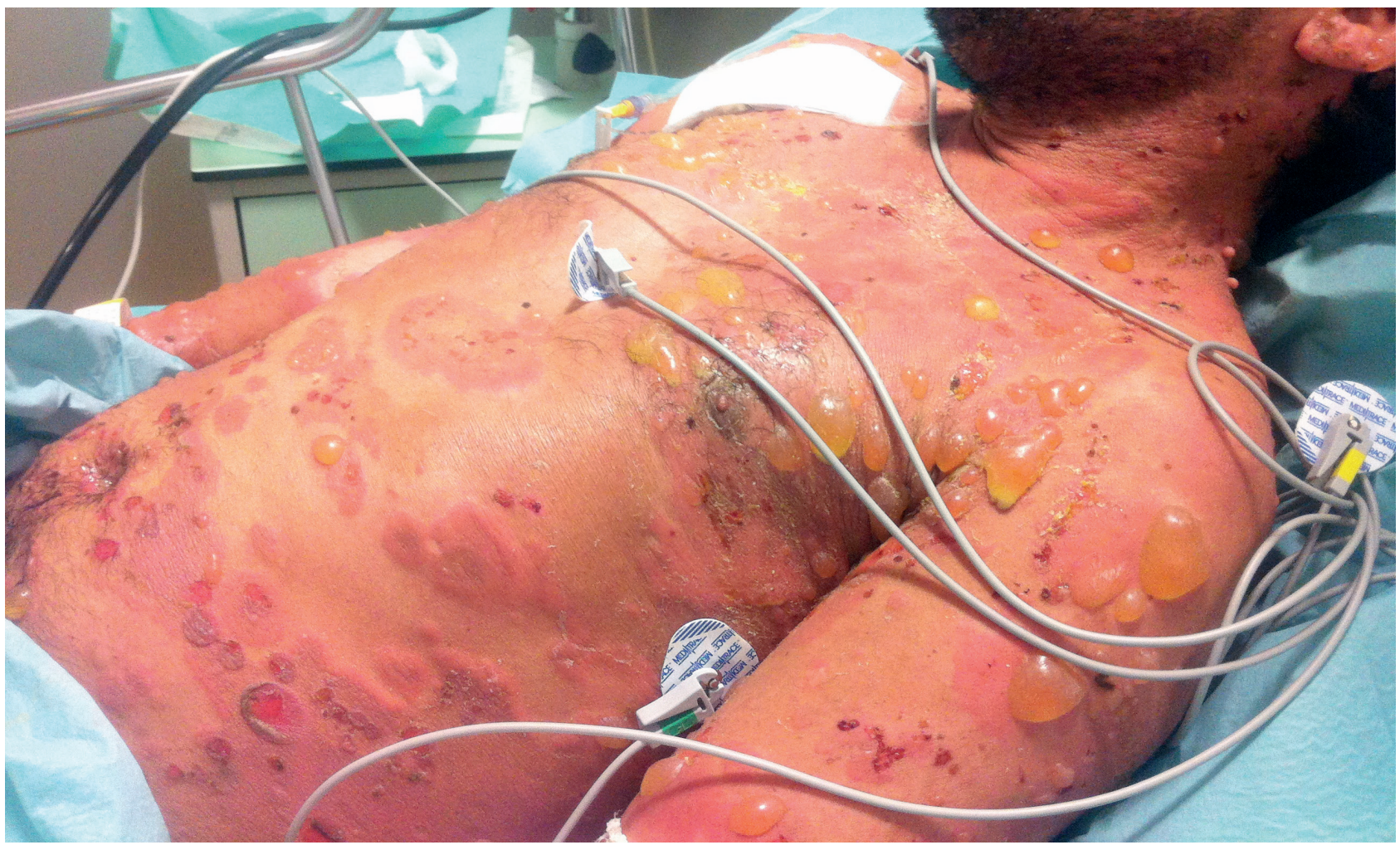

Figura 1 - Antes de iniciar tratamento

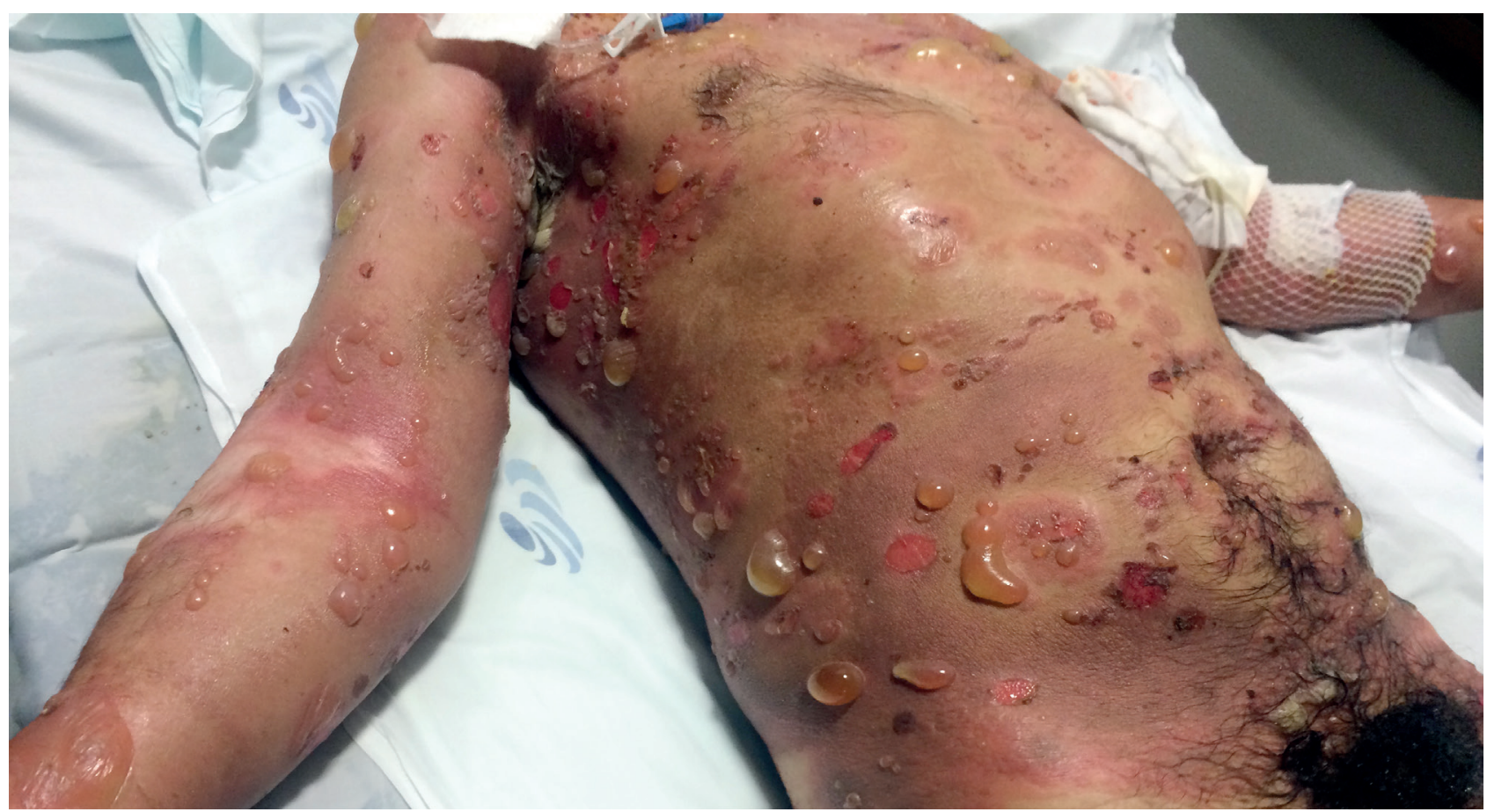

Figura 2 - Depois de iniciar tratamento convencional e antes de iniciar rituximab

\section{DISCUSSÃO}

O penfigoide bolhoso é uma doença autoimune caracterizada pela formação de bolhas subepidérmicas. $\mathrm{O}$ mecanismo é mediado por autoanticorpos direcionados contra duas proteínas (BP180 e BP320) presentes no complexo de adesão entre a derme e a epiderme. Clinicamente manifesta-se por bolhas tensas, com diâmetro entre $1 \mathrm{~cm}$ e 3 $\mathrm{cm}$, distribuídas normalmente pelo tronco, membros e pre- gas axilares e inguinais, também podem afetar as mucosas. As bolhas podem ser precedidas ou associadas a prurido, por vezes intenso, e placas urticariformes. ${ }^{2} \mathrm{O}$ diagnóstico neste doente baseou-se na biópsia cutânea e na presença dos anticorpos típicos desta entidade, sendo que o gold standard para o diagnóstico é a avaliação por imunoflurescência direta de tecido cutâneo afetado. ${ }^{1}$ Está bem estabelecida a associação entre o PB e doenças neurológicas 


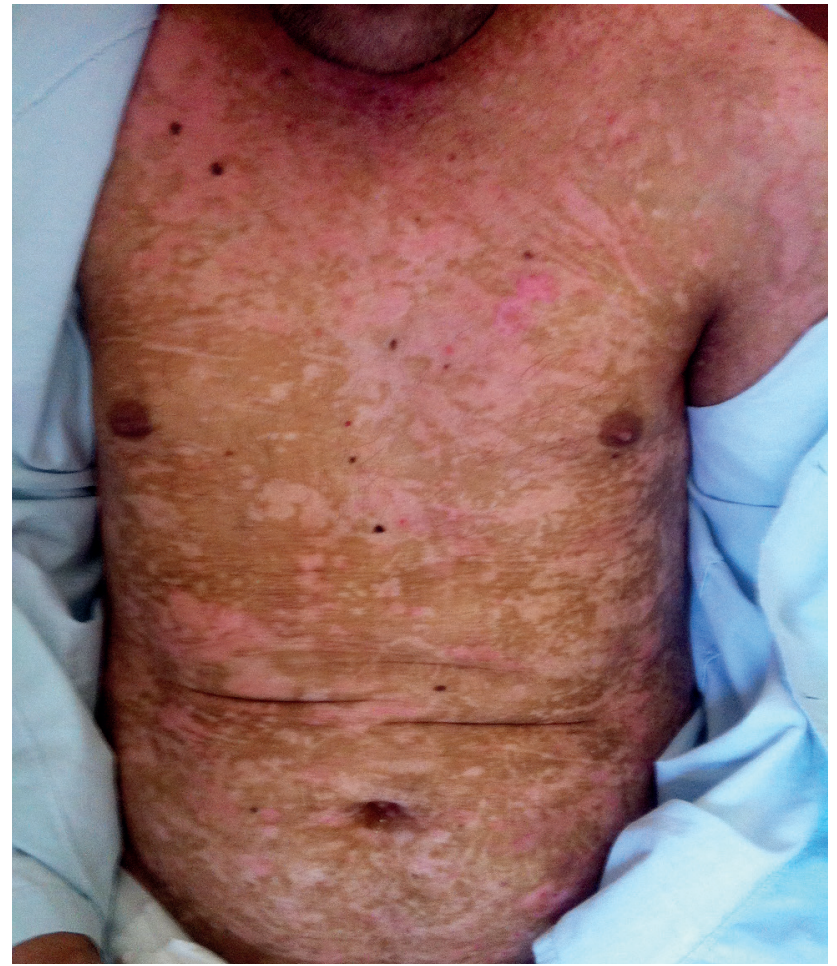

Figura 3 - Após 4 ciclos de rituximab

como demência, doença de Parkinson, doença bipolar. Apesar de poder ser uma doença autolimitada, o desafio neste doente foi conseguir controlar a doença, que nos períodos de atividade é responsável por grande morbilidade e mortalidade. A grande maioria dos casos é bem controlada com o uso de corticoides tópicos ou sistémicos, sendo que a dose de PDN pode variar entre 0,5 a $1 \mathrm{mg} / \mathrm{kg}$ dependendo da gravidade e extensão da doença. Nos casos em que a corticoterapia por si só não é eficaz estão indicados outros imunossupressores como AZA, micofenolato mofetil e metotrexato. A IgIV é universalmente usada como imunomodelador e estão descritos casos de sucesso em casos de PB, isoladamente ou em associação a outros fármacos. ${ }^{4}$ Neste doente apesar da escalada terapêutica com três tratamentos comprovadamente eficazes em simultâneo a doença mantinha-se muito ativa.

Devido à gravidade e refratariedade ao tratamento instituído decidiu-se iniciar rituximab com excelente resposta. O rituximab, um anticorpo monoclonal anti CD-20, é capaz de induzir rápida e eficazmente a depleção dos linfócitos $B$ CD20+ responsáveis pela produção dos anticorpos envolvidos no penfigoide bolhoso. Além disso permite a formação de uma linhagem naive de linfócitos $B$ o que explica o seu efeito prolongado. ${ }^{5}$ Atualmente o doente encontra-se sem atividade da doença e com evolução clínica e laboratorial favorável.

Existem poucos casos relatados de PB tratado com biológicos mas uma meta-análise identificou 16 doentes com PB tratados com rituximab em que 69\% tiveram resposta completa. Nessa amostra, um doente não obteve resposta e três acabaram por falecer. ${ }^{6}$

O regime terapêutico ideal com rituximab ainda não

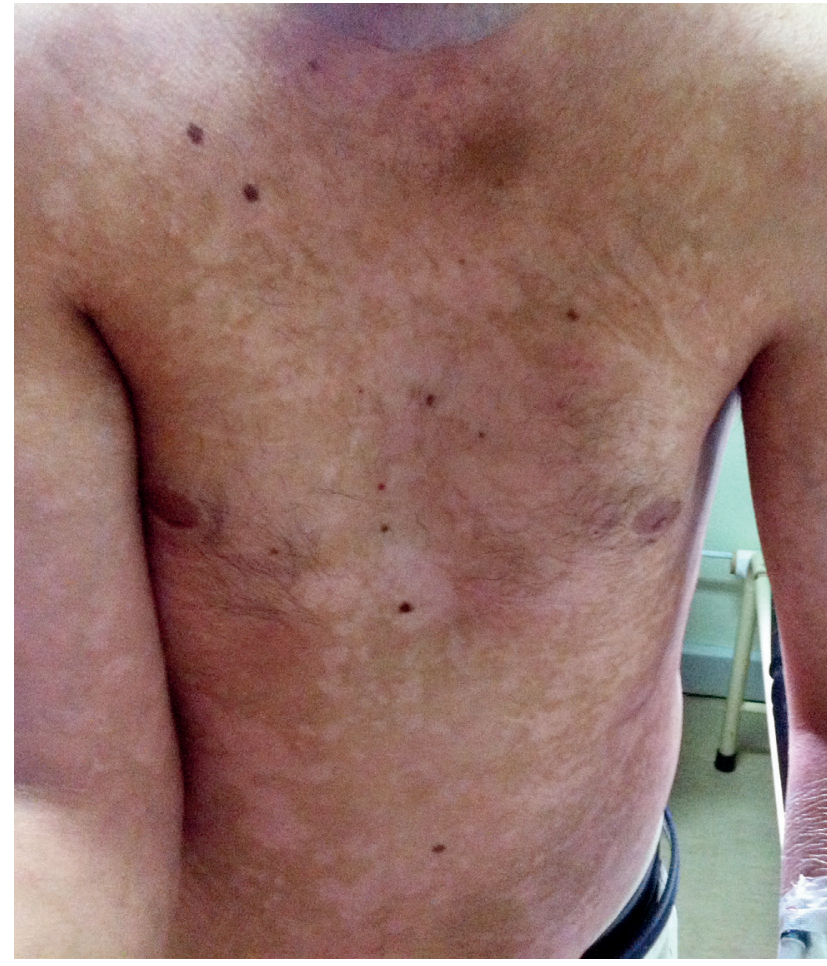

Figura 4 - Dezasseis semanas após tratamento com rituximab

está bem definido e existem vários protocolos propostos. Uma meta-análise comparou a eficácia dos diferentes regimes com rituximab no tratamento do pênfigo, foram analisados 578 doentes incluídos em 30 estudos. Observou-se remissão completa em $76 \%$ dos doentes após um ciclo de tratamento e a média de tempo até controlo da doença foi inferior a um mês. Tratamentos com doses maiores de rituximab estiveram associados a períodos maiores de remissão da doença. ${ }^{7}$

Outro estudo comparou o outcome de doente tratados com PDN ou com PDN mais rituximab e observou-se meIhor controlo da doença, menor dose de PDN para controlo da doença e menor mortalidade no grupo tratado com terapêutica combinada. ${ }^{8}$

O rituximab tem um bom perfil de segurança e os seus efeitos secundários mais frequentes são náuseas, vómitos, urticária e tosse, que normalmente são ligeiros e autolimitados. Contudo é importante não esquecer que este tratamento pode ter efeitos secundários graves e fatais, nomeadamente as infeções. Assim, a sua utilização implica a exclusão de infeções latentes, como hepatites e tuberculose, e vigilância apertada para o aparecimento de infeções oportunistas. ${ }^{5}$ Apesar da boa eficácia do rituximab as recidivas podem ocorrer e nessas situações deve-se ponderar repetir o tratamento. ${ }^{9}$

O tratamento nos casos mais graves é prolongado, com desmame de corticoides, associado ou não a outro imunossupressor, que pode ir de quatro a 10 meses. A monitorização é feita através de avaliação clínica e doseamentos seriados do anti-BP180, cujos níveis estão correlacionados com a actividade da doença. ${ }^{1}$ Para a avaliação clínica podem ser usadas escalas como o BPAI (Bullous Pemphigoid 
Disease Area Index), o PDAI (Pemphigus Disease Area Index) ou o MMPDAI (Mucous Membrane Pemphigoid Disease Area Index), que permitem uma avaliação mais objetiva das lesões e caracterizar melhor a sua evolução. ${ }^{10}$

\section{CONCLUSÃO}

O rituximab é cada vez mais usado em doenças mediadas por autoanticorpos. Apesar de haver poucos casos descritos do uso de rituximab no penfigoide bolhoso, a grande maioria são situações de sucesso e com resposta sustentada, como neste doente. Assim, os autores pretendem relembrar que este fármaco deve ser considerado em casos de penfigoide bolhoso refratários ao tratamento convencional ou mesmo como primeira linha nos casos mais agressivos.

\section{REFERÊNCIAS}

1. Fuertes de Vega I, Iranzo-Fernández P, Mascaró-Galy JM. Bullous pemphigoid: clinical practice guidelines. Actas Dermosifiliogr. 2014;105:328-46

2. Kasperkiewicz M, Zillikens D. The pathophysiology of bullous pemphigoid. Clin Rev Allerg Immunol. 2007;33:67-77.

3. Lever WF. Pemphigus. Medicine. 1953;32:2-123.

4. Venning VA, Taghipour K, Mohd Mustapa MF, Highet AS, Kirtschig G. British Association of Dermatologists' guidelines for the management of bullous pemphigoid 2012. Br J Dermatol. 2012;167:1200-14.

5. Joly P, Mouquet H, Roujeau JC, D'Incan M, Gilbert D, Jacquot S, et al. A single cycle of rituximab for the treatment of severe pemphigus. $\mathrm{N}$ Engl J Med. 2007;357:545-52.

6. Shetty S, Ahmed AR. Treatment of bullous pemphigoid with rituximab: critical analysis of the current literature. J Drugs Dermatol. 2013;12:672-7.

\section{AGRADECIMENTOS}

Os autores gostariam de agradecer a Trigo Faria e Francisco Esteves pela sua colaboração neste caso.

\section{CONFLITOS DE INTERESSE}

Os autores declaram não terem qualquer conflito de interesse relativamente ao presente artigo.

\section{FONTES DE FINANCIAMENTO}

Os autores declaram não ter recebido subsídios ou bolsas para a elaboração do artigo.

7. Wang $\mathrm{HH}$, Liu CW, Li YC, Huang YC. Efficacy of rituximab for penphigus: A systematic review and met-analysis of different regimens. Acta Derm Venereol. 2015;95:928-32.

8. Cho YT, Chu CY, Wang LF. First-line combination therapy with rituximab and corticosteroids provides a high complete remission rate in moderateto-severe bullous pemphigoid. Br J Dermatol. 2015;173:302-4.

9. Kasperkiewicz M, Shimanovich I, Ludwig RJ, Rose C, Zillikens D, Schmidt E. Rituximab for treatment-refractory pemphigus and pemphigoid: A case series of 17 patients. J Am Acad Dermatol. 2011;65:552-8.

10. Murrell DF, Marinovic B, Caux F, Prost C, Ahmed R, Wozniak K, et al. Definitions and outcome measures for mucous membrane pemphigoid: Recommmendations of an international panel of experts. J Am Acad Dermatol. 2015;72:168-74.
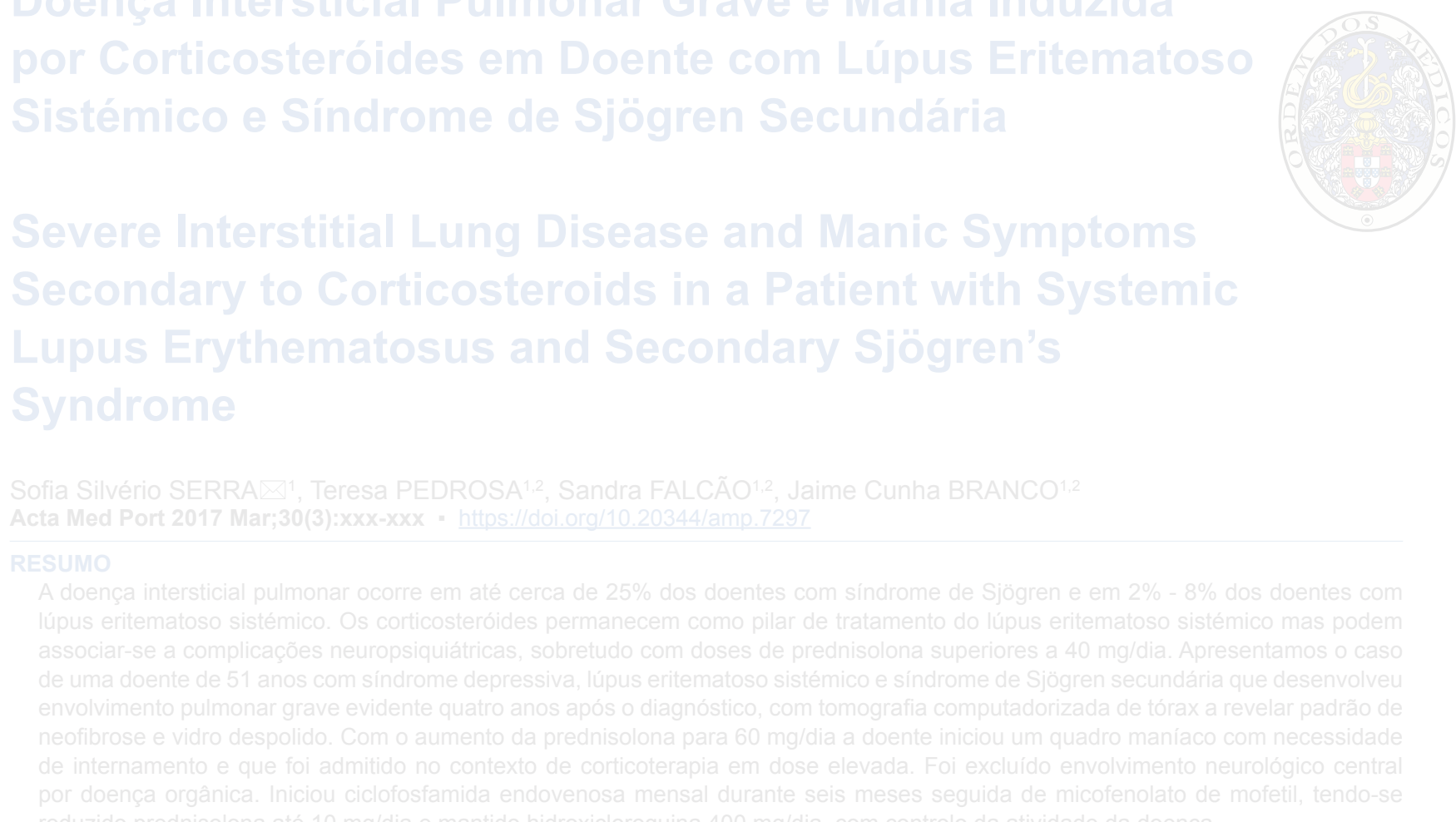